\title{
DIAGNÓSTICO DE LA GENERACION Y MANEJO DE RESIDUOS SÓLIDOS EN LA UNIVERSIDAD DEL QUINDÍO
}

\author{
DIAGNOSIS OF GENERATION AND HANDLING \\ OF SOLID RESIDUALS IN THE UNIVERSITY OF QUINDÍO
}

Olga Alicia Nieto C., Margarita Marcela Nieto R., Carlos Mario Lozano, Luis Fernando Jiménez.

Centro de Investigaciones Biomédicas, Facultad Ciencias de la Salud, Universidad del Quindío.

Fecha de recibido: Febrero 3 de 2010

Fecha de aceptado: Junio 9 de 2010

Correspondencia: Programa de Medicina, Universidad del Quindío, Av. Bolívar calle 12 norte Armenia Quindío.

Correo electrónico: olgalicianieto@gmail.com

\section{RESUMEN}

La generación de residuos sólidos es uno de los principales problemas de salud y ambiente, que evidencia el uso que hacemos de este planeta y la huella ecológica que dejamos en él.

En Colombia, se estima que a nivel nacional se generan aproximadamente 30.886 toneladas diarias, el departamento de Quindío 531 ton/día de residuos sólidos, de los cuales Armenia representa el 37\% (196 ton/día).

La generación de residuos sólidos en la Universidad del Quindío es de $210 \mathrm{Kg} / \mathrm{día}$. De los cuales 16,8 kg (8\%) son biodegradables u orgánicos, el 3,4\% (7,2 kg) son reciclables (papel), el mayor volumen son ordinarios (incluye inertes), $182 \mathrm{Kg}$ que equivalen al 86,66\%. Peligrosos (biosanitarios) son 3,8 $\mathrm{Kg}(1,9 \%)$ Es decir se generan entre 63 a 76 toneladas de residuos sólidos al año.

La generación per cápita, es de 23,33 gr/día. En un año es de 8 a 9,5 kg per cápita. Se calculó identificando la población universitaria en 8.000 personas.

El objetivo de este proyecto fue elaborar el diagnóstico de la generación y manejo de residuos sólidos en la Universidad del Quindío, con el propósito de diseñary proponer en funcionamiento un Sistema de Gestión Ambiental.

Simultáneamente con este proceso, se puso en funcionamiento el Sistema de Gestión Ambiental en la Universidad del Quindío, con participación de diversos estamentos y dependencias de la Universidad.

Palabras claves: Manejo de residuos sólidos, sistema de gestión ambiental.

\section{ABSTRACT}

The generation of solid residuals is one of the main environmental and health problems, it evidences the use that we make of this planet and the ecological print that we leave in it. In Colombia, it is considered that at national level are generated 30.886 daily tons approximately, the department of Quindio 531 tons per day of solid residuals, of which Armenia represents $37 \%$ (196 tons per day). The generation of solid residuals in the University of Quindio is of $210 \mathrm{Kg}$ per day, of which 16,8 $\mathrm{kg}(8 \%)$ are biodegradable or organic, 3,4\% (7,2 kg) are recyclable (paper), the biggest volume is ordinary (it includes inert), $182 \mathrm{Kg}$ that are equal to $86,66 \%$, dangerous (bio-sanitary) they are $3,8 \mathrm{Kg}(1,9 \%)$. That is to say there are generated up to 63 to 76 tons of solid residuals per year. The generation of solid residuals per capita is 23,33 gr per day. In one year it is up to 8 to 9,5 kg per capita, this was calculated identifying the university population in 8.000 people.

The objective of this project was to elaborate the diagnosis of the generation and handling of solid residuals in the University of Quindio, with the purpose of design and propose a System for Environmental Administration.

Keywords: Solid residuals, solid residuals management, system of environmental administration.

\section{INTRODUCCION}

En la Universidad del Quindío se generan diariamente residuos sólidos, líquidos y gaseosos, pero se desconoce a cuanto asciende la generación y no existe un manejo integral de residuos.

Si bien existe algún aprovechamiento y reciclaje por algunos funcionarios de la Universidad de los residuos sólidos (papel), esto ocurre de manera fragmentada, sin un enfoque sistémico.
Hasta el momento se han instalado canecas para clasificar los residuos sólidos, con base en un código de colores y se está diseñando una campaña educativa entre la ARP, Comunicación y Sistemas. Estos esfuerzos de la ARP han sido productivos, pero se llevan a cabo dentro de los alcances y limitaciones que implican su quehacer como administradora de riesgos profesionales.

Se ve como una limitante los recursos necesarios para montar el Sistema de Gestión de Residuos y no como una 
oportunidad de generación de alternativas ambientales, de empleo y de negocio. Y no existe aún el diagnóstico integrador con miras al diseño de un Sistema de Gestión Integral de Residuos para la Universidad.

Existe un marco normativo vigente, constituido principalmente por el Decreto 2676 de 2000, su reglamentación y manuales correspondientes, expedidos por el Ministerio de Salud y el de Ambiente en conjunto.

La Universidad está en mora de diseñar y poner en funcionamiento un Sistema de Gestión Integral de Residuos, mediante el cual :

- Se reutilicen los materiales

- Se reciclen los que no es posible reutilizar

- Se conviertan los residuos sólidos generados diariamente en abono orgánico.

- Iniciar el manejo de los residuos líquidos, para evitar la contaminación de la Microcuenca La Aldana y las Quebradas del Municipio, que finalmente van al Rio Quindío y la Río La Vieja.

Todo esto puede lograrse a través de un Sistema de Gestión Integral de Residuos, que además de minimizar impactos ambientales, generará empleo y subproductos útiles al medio universitario y a la sociedad.

El alcance del presente proyecto de investigación es un aporte descriptivo de la situación actual, que permita avanzar en una segunda fase en el diseño y puesta en funcionamiento de un Sistema de Gestión Ambiental de Residuos en la Universidad del Quindío.

\section{MATERIALES Y MÉTODOS}

Se llevó a cabo un estudio descriptivo de corte transversal de los residuos generados en la Universidad del Quindío, su manejo y su impacto ambiental.

La población la constituyen las diferentes dependencias de la Universidad del Quindío y los residuos que se generan en cada una de ellas.

Se hizo una descripción de los residuos sólidos que se generan en la Universidad, se tuvieron en cuenta las etapas comprendidas desde su emisión, acopio, hasta su disposición final.

\section{Recolección de información}

Se hizo acompañamiento al personal de aseo en su horario de trabajo (entre las cinco y las once de la mañana), en todos los edificios que componen el campus universitario para identificar la primera recolección de los residuos en cada uno de los edificios de la Universidad.

Se hizo seguimiento a la persona encargada de los servicios generales y se dibujó en un mapa su recorrido y los tiempos de barrido y limpieza.

- Se ubicaron los recipientes para los residuos

- Se pesaron los residuos que salen de esa área en un día

- Se tomó registro fotográfico de los residuos

- Se describieron los residuos

- Se describió la clasificación como : adecuada, regular o inadecuada.

- Se hizo seguimiento de la ruta de recolección hasta el sitio de disposición en la universidad y definición de microrutas.

- Se registró la información.

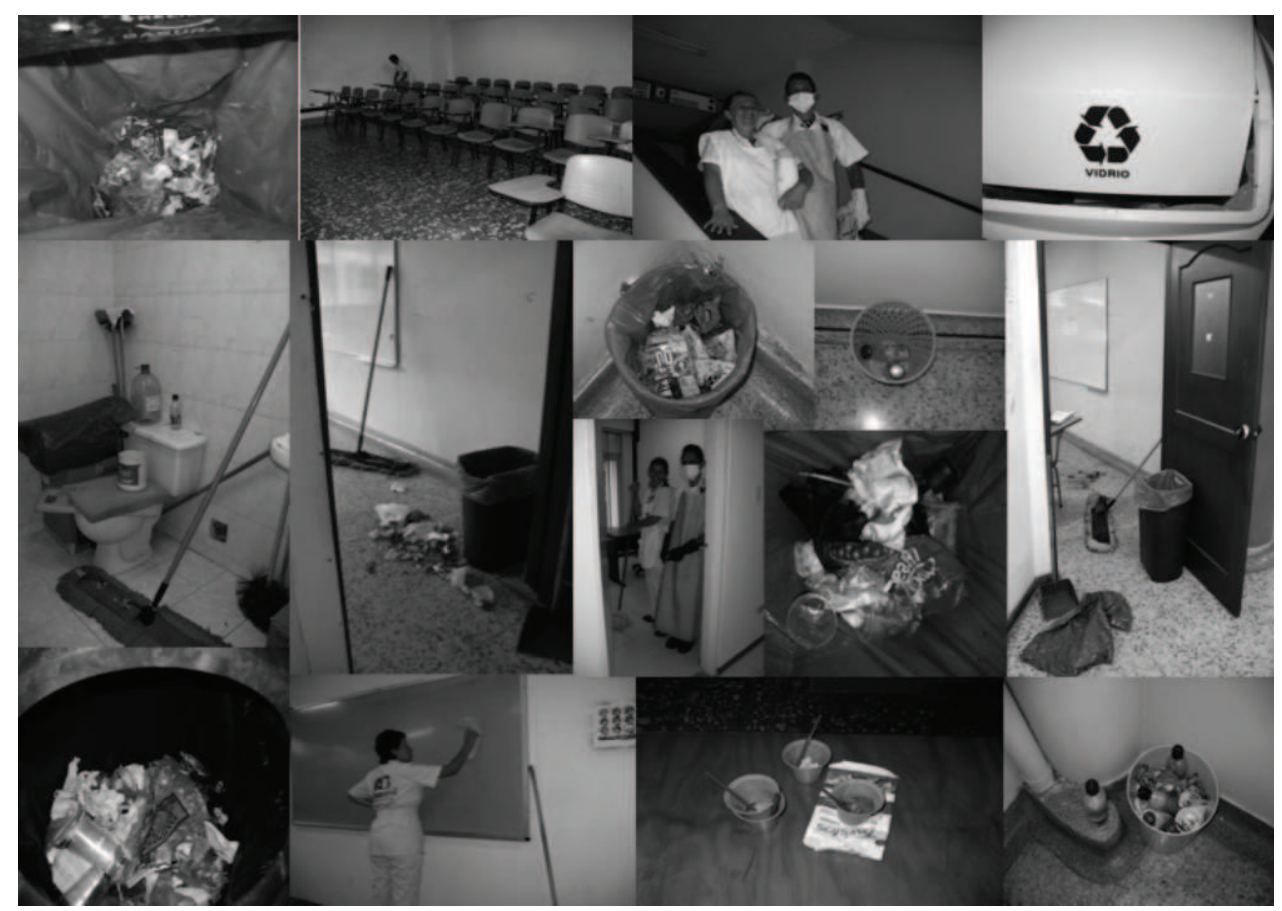

Figura 1. Recolección de información en el recorrido por cada dependencia.

Rev. Invest. Univ. Quindío (20): 153 - 165. Armenia - Colombia 
Sistematización de la información para el análisis en equipo de trabajo

- El equipo de trabajo se reunió semanalmente para la sistematización y análisis de la información, con los respectivos informes de avance.

- Se organizó la información en un archivo de excel .xls para alimentar la base de datos diseñada en el programa SPSS con las variables del formato de recolección de información.
- Se realizaron los cálculos de las áreas de limpieza y longitud de barrido en los planos por dependencia, con el dibujo de los recorridos de cada persona de servicios generales. Y se alimentó la base de datos en SPSS.

- Se diseñaron las consultas a la base de datos y se realizaron los análisis.

- A continuación se presenta la metodología llevada a cabo en un flujograma resumen.

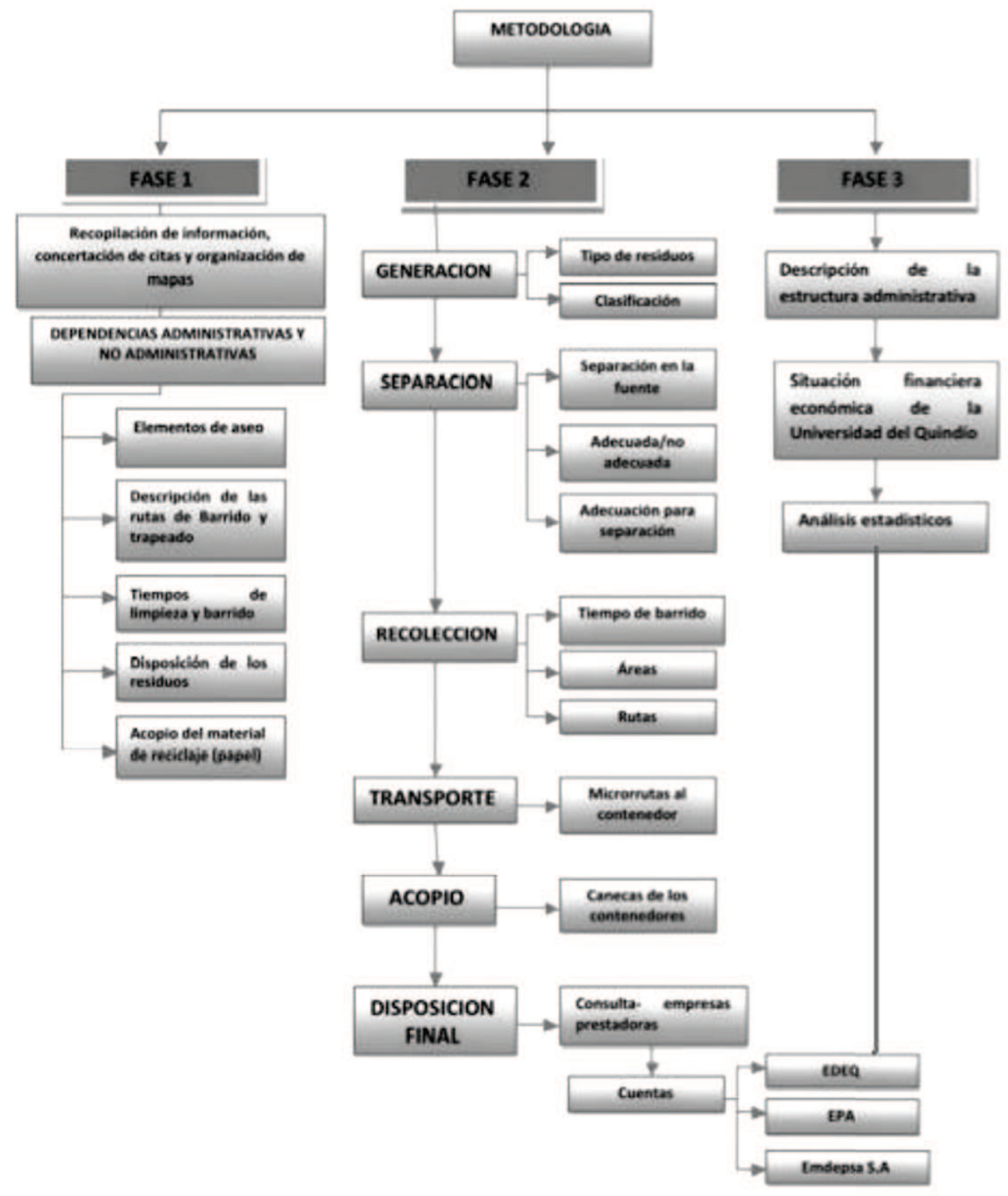

Figura 2 : Flujograma de la metodología. 


\section{RESULTADOS}

Generación de residuos sólidos día/mes/año.

Para un día de jornada habitual en la universidad se calculó una generación diaria de $\mathbf{2 1 0 , 7}$ kilos, este peso tiene en cuenta todos los residuos sólidos : biodegradables u orgánicos, reciclables (entre ellos el papel), inertes, ordinarios y peligrosos (biosanitarios). Se calcula una generación a la semana entre $1.264,3$ y 1.475,01 kilos; al mes entre $5.057,20$ y $6.321,51$ kilos y al año entre $63.215,1$ y $75.858,12$ kilos. Es decir en la Universidad se generan entre 63 a 76 toneladas de residuos sólidos en el año.

Estos residuos no se compactan, se depositan en los contenedores directamente para la recolección por Servigenerales.

Los residuos biodegradables $u$ orgánicos se segregan en la fuente, especialmente en el restaurante y constituyen el $8 \%$ del total de los residuos de la universidad : 16,8 kilos en un día promedio, lo cual representa un $31,53 \%$ del total de los residuos que se generan allí $(53,127 \mathrm{~kg})$. En el año corresponden aproximadamente entre 5 y 6 toneladas año de residuos orgánicos. A pesar de la segregación en la fuente con la intención de llevarlos a la granja para producir compost, se recogen rápidamente y se juntan con los demás residuos en los contenedores.

La generación de los residuos peligrosos (biosanitarios y cortopunzantes) asciende a $1,9 \%$ - 3,8 kilos, respectivamente, los cuales se originan en medicina, bienestar universitario y biomédicas.

Los residuos sólidos peligrosos, químicos, son generados por el laboratorio de aguas y el de suelos, debidamente inactivados, para su disposición final. No se generan residuos radioactivos en la Universidad.

El manejo de los residuos peligrosos biosanitarios es adecuado, en estas dependencias, según el marco normativo del Ministerio de Salud, se depositan en guardianes y en bolsas rojas debidamente marcadas. Y son recogidos hasta un sitio de disposición intermedia ubicado en biomédicas, para su recolección por Emdepsa.

Generación de residuos sólidos por dependencia, al día/mes/año.

El gráfico permite identificar que las dependencias que presentan mayor generación de residuos sólidos son: Ciencias básicas con $18 \%$ que corresponde a un peso de $37,69 \mathrm{~kg}$; Restaurante con $25 \%$ equivalente $53,127 \mathrm{~kg}$; Publicaciones $8,20 \%$ equivalente $17,26 \mathrm{~kg}$; Coliseo, Cafetería canchas y Piscinas 6,32\% equivalente 13,32 kg; Archivo 5,10\% equivalente 10,76 kg; Agroindustria 5,51\% equivalente 11,63 kg; Educación 4,42\% equivalente 9,327 kg; Ingeniería 4,17\% equivalente 8,79 Kg; y Medicina 3,77\% equivalente $7,943 \mathrm{Kg}$.

La recolección del papel no se hace diariamente, sino que varía de acuerdo a la producción por cada dependencia. Se acopia inicialmente por el personal de servicios generales en las cocinetas o baños; cuando existe un volumen adecuado, se recoge y lleva a otro sitio de acopio, por el personal de Telesai, Sin embargo, algunas dependencias lo reciclan independientemente, tales como Ingeniería, Planeación, algunos administrativos y los recursos de la venta del papel se utilizan para algunos gastos cuando se trabaja en horas extras. Se pesó el papel acopiado en un periodo de tiempo equivalente a $216 \mathrm{~kg} / \mathrm{mes}$ y se dividió por el número de días, calculando una generación promedio día de 7,2 kg.

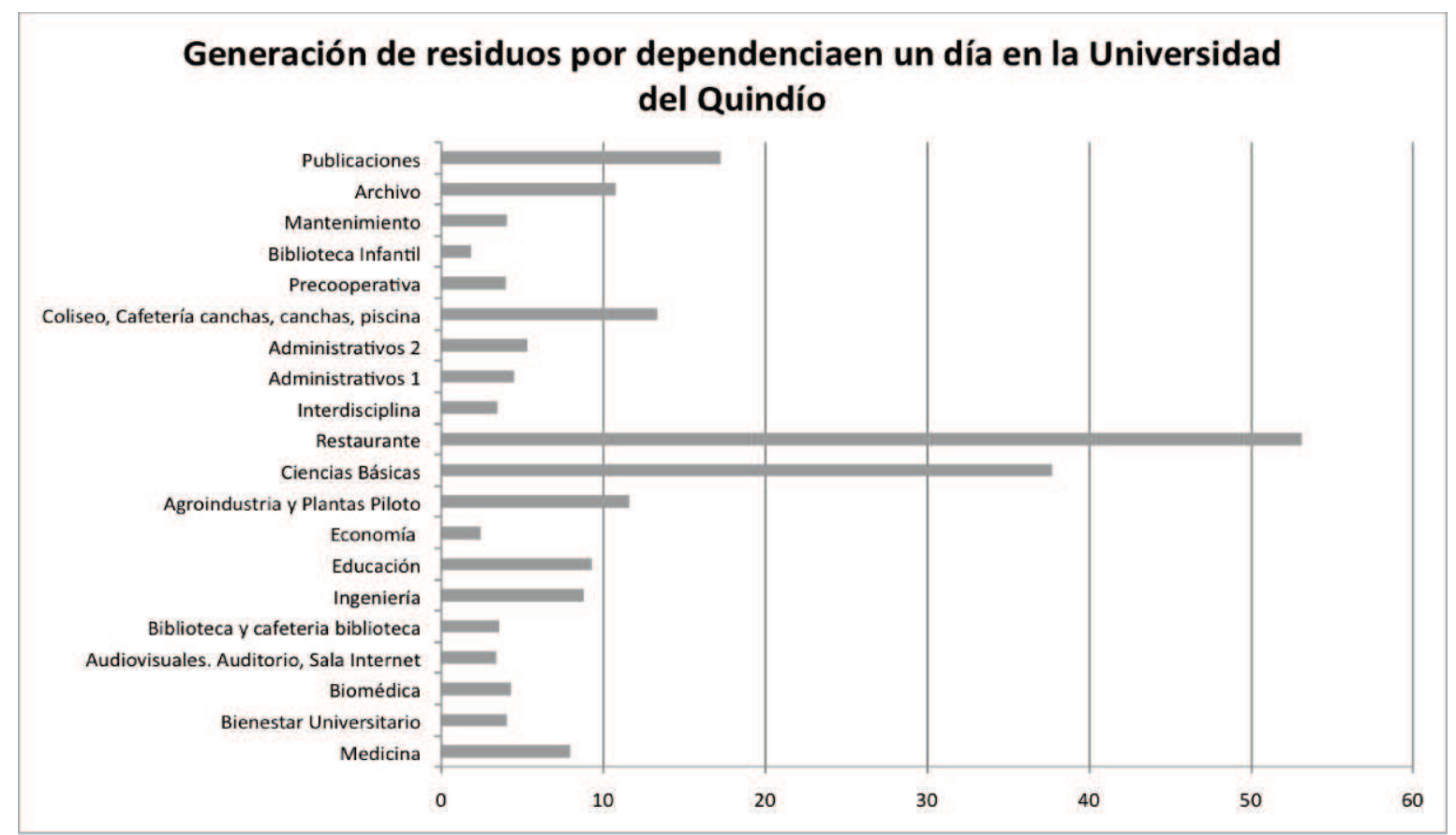

Figura 3. Generación de residuos por dependencia (kg/día)

La mayor generación en $\mathrm{Kg} /$ día corresponde al restaurante, ciencias básicas y publicaciones 
Tipo de residuos sólidos generados.

De acuerdo a la clasificación de residuos propuesta por el Decreto 2676 del 2000 se presenta a continuación la tipología de residuos sólidos que se genera en la universidad.

Tabla 1. Clasificación de residuos

RESIDUOS NO PELIGROSOS RESIDUOS PELIGROSOS

$\begin{array}{ll}\text { BIODEGRADABLES U ORGANICOS } & \text { QUIMICOS } \\ \text { RECICLABLES } & \text { INFECCIOSOS } \\ \text { INERTES } & \text { RADIOACTIVOS }\end{array}$

ORDINARIOS

Se presentan las fotos que nos ilustran la situación encontrada para cada una de las categorías establecidas.

\section{TIPO DE RESIDUOS (NO PELIGROSOS): BIODEGRADABLES}

Productos naturales que se descomponen fácilmente. Entre ellos residuos alimenticios, madera y demás transformables en materia orgánica.

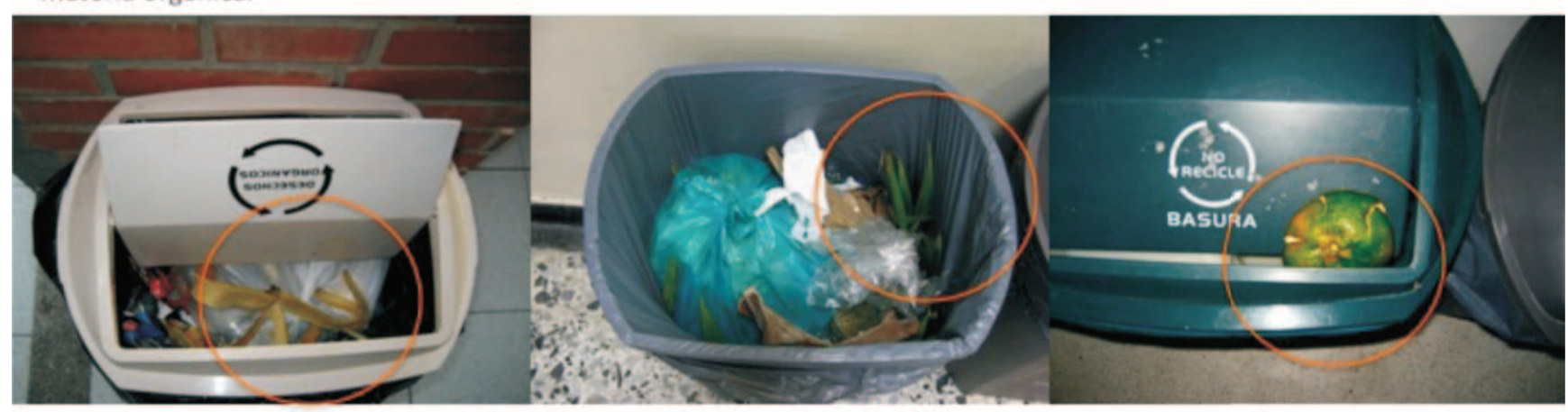

Ciencias de la salud

Administrativo 1

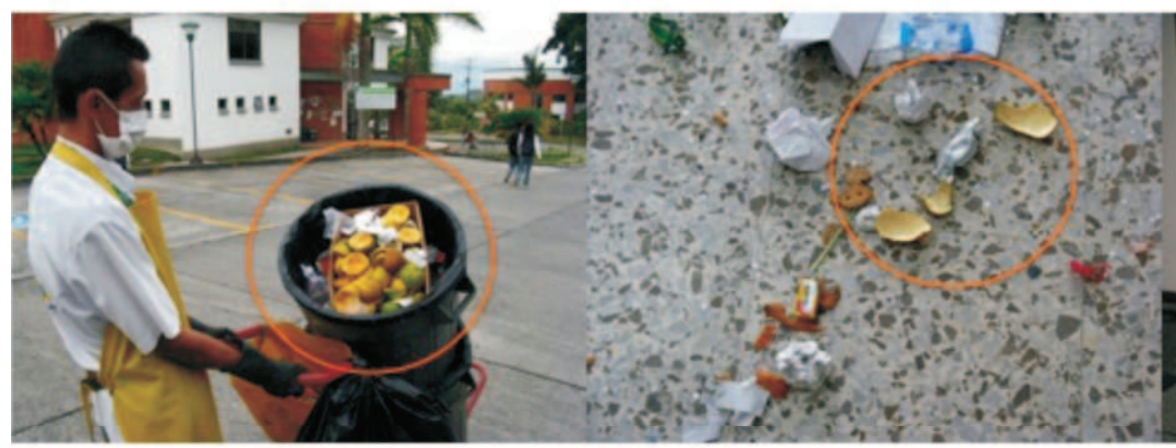

Puesto de jugos Medicina-Biblioteca

Educación

Figura 4. Residuos sólidos no peligrosos - Biodegradables.
Administrativo 2

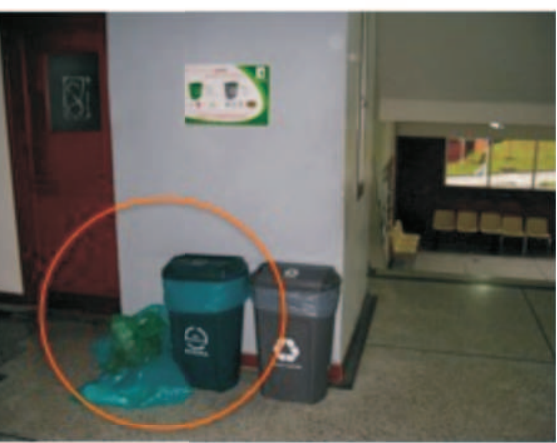

Ingenieria. Separación en la fuente: Verde - Biodegradables 


\section{TIPO DE RESIDUOS (NO PELIGROSOS): RECICLABLES}

Materiales que no se descomponen fácilmente, sin embargo, se conocen procesos en los cuáles pueden ser transformados y utilizados como materia prima para nuevos productos.
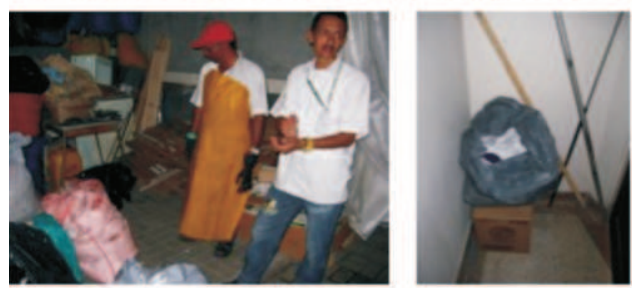

Acopio de papel en el sótano de Ciencias Básicas

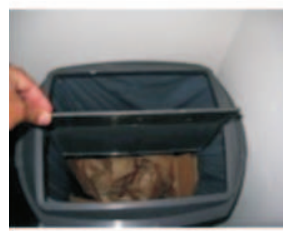

Ingenieria

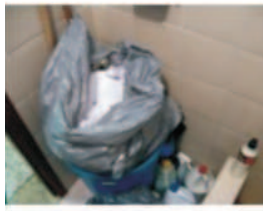

Educación
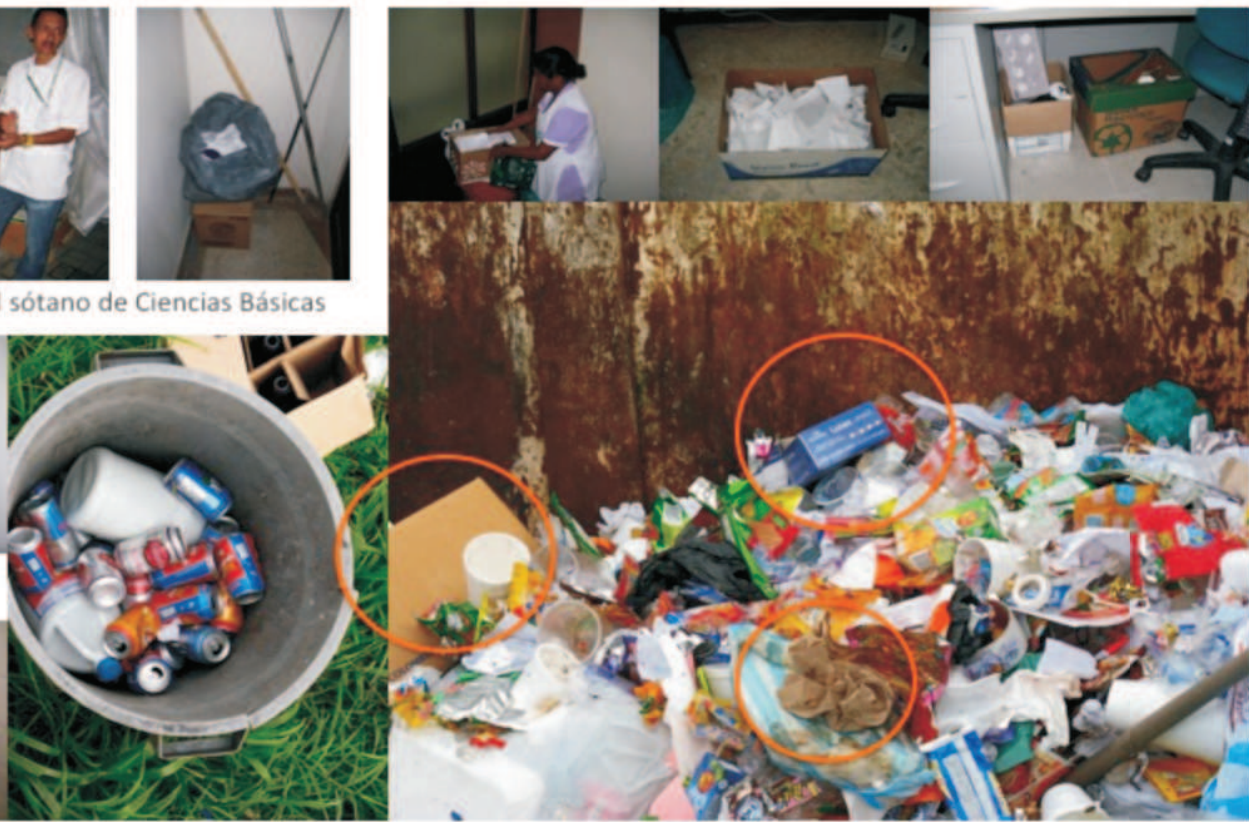

CONTENEDOR - En el parqueadero de Ingenieria

Figura 5. Residuos sólidos no peligrosos - Reciclables.

Sólo una pequeña parte de los residuos reciclables (papel, plástico) se recupera para la venta. Los demás terminan en la góndola de disposición como residuos sólidos ordinarios.

\section{TIPO DE RESIDUOS (NO PELIGROSOS): INERTES}

Materiales que no son reutilizables o reciclables y requieren largos períodos de descomposición. Algunos son: el icopor y ciertos tipos de plásticos.

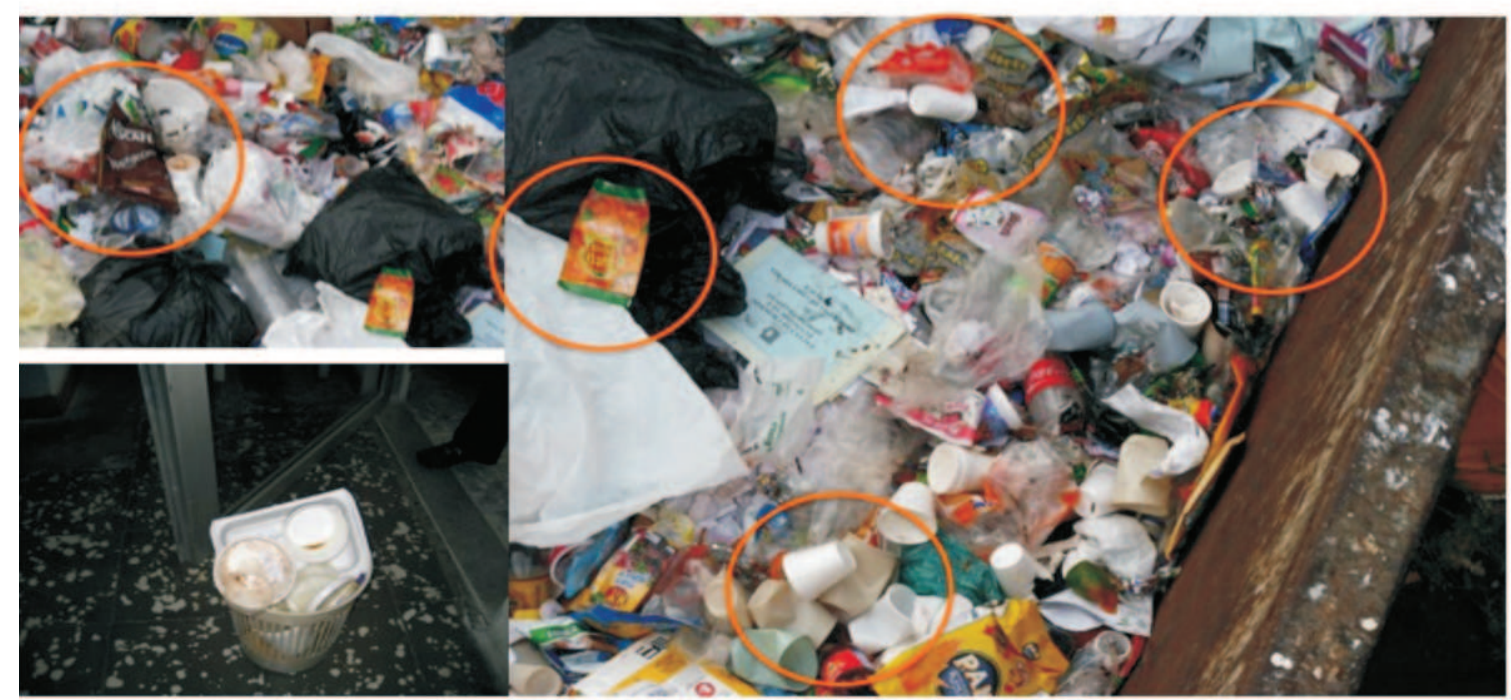

Ingenieria

CONTENEDOR - En el parqueadero de Ingenieria

Figura 6. Residuos sólidos no peligrosos - Inertes.

Rev. Invest. Univ. Quindío (20): 153 - 165. Armenia - Colombia 
Los residuos sólidos inertes, como el icopor y algunos plásticos, terminan en la góndola de disposición como residuos sólidos ordinarios.

\section{TIPO DE RESIDUOS (NO PELIGROSOS): ORDINARIO}

Materiales comúnmente generados en la Universidad que no son aptos o valorados para reciclaje: cartón plastificado, vidrio, plástico, metal no recuperable, servilletas, residuos sanitarios, residuos tecnológicos (electrodomésticos, equipos, baterias)

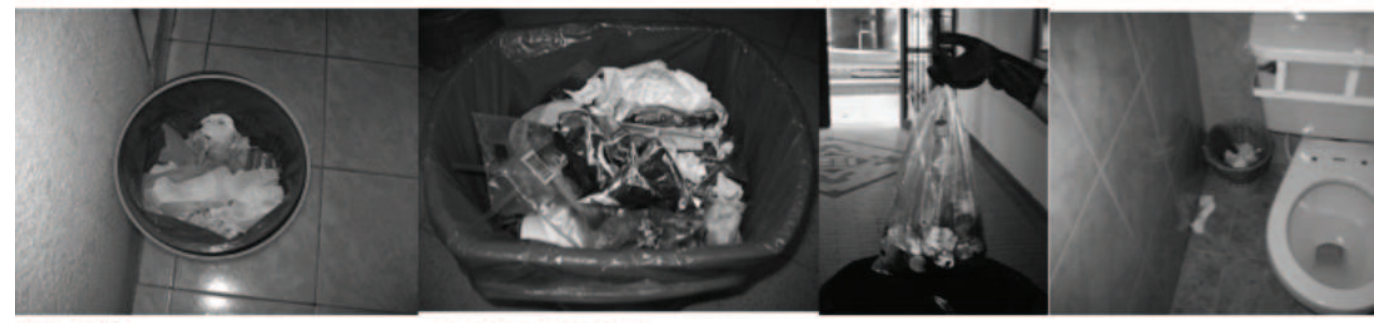

Ingenieria

Ciencias de la salud

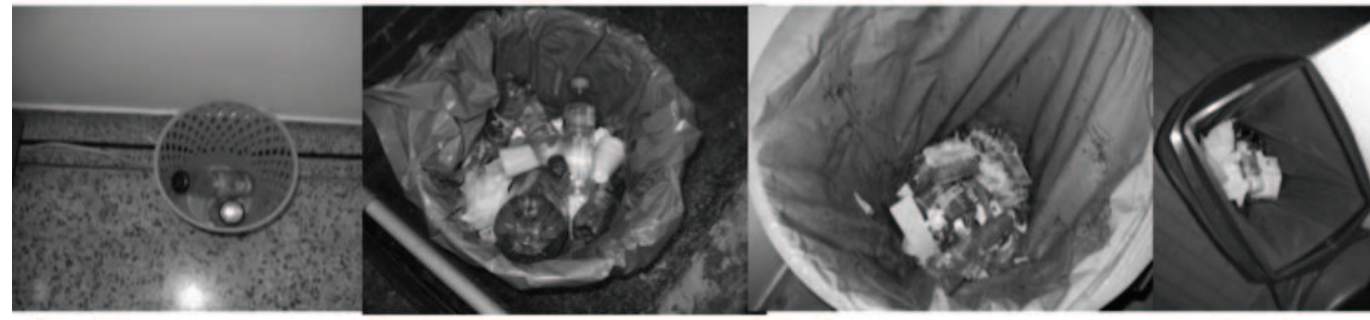

Educación

Plantas piloto

Ciencias básicas

Audiovisuales

Figura 7. Residuos sólidos no peligrosos - Ordinarios

Los residuos se juntan finalmente, a pesar de su segregación inicial, como residuos sólidos ordinarios, para la recolección por Servigenerales.

TIPO DE RESIDUOS (PELIGROSOS): BIOSANITARIOS Y CORTOPUNZANTES
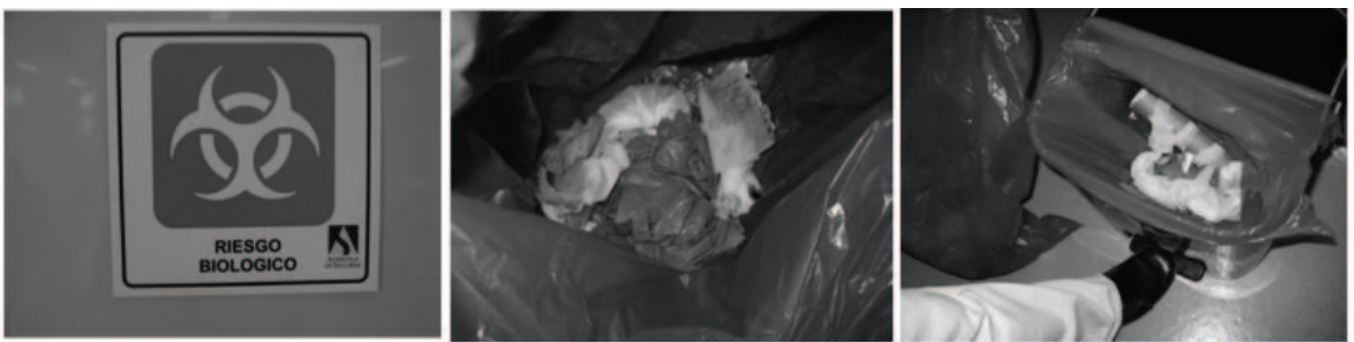

Biomédicas

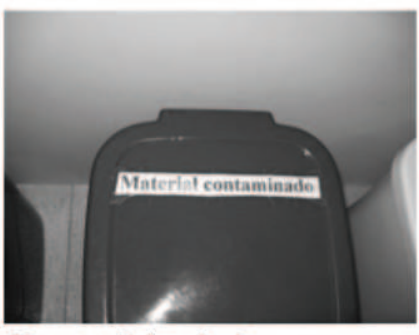

Bienestar Universitario
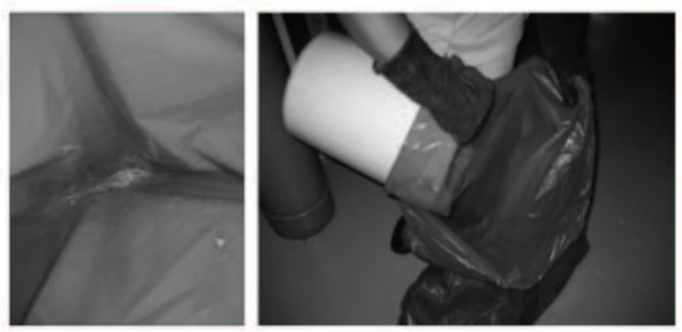

Ciencias de la salud

Figura 8. Residuos sólidos peligrosos - Biosanitarios y cortopunzantes 


\section{Tipo de residuos peligrosos : químicos}

Si bien, el presente estudio tiene como objeto los residuos sólidos, es de resaltar el excelente manejo de los residuos químicos en los Laboratorio de Aguas y de Suelos, en donde desde su diseño inicial se neutralizan los ácidos y posteriormente se disponen en un sitio para ello.

Los otros laboratorios acumulan sus residuos químicos en frascos clasificados y la Universidad debe identificar la magnitud de este problema y definir el manejo de los residuos, que se acumulan allí.

\section{Tipo de residuos peligrosos : radioactivos}

No se tiene información sobre residuos radioactivos en la Universidad del Quindío.

\section{Generación per cápita}

Cálculo de la población presencial universitaria :

Para el cálculo de la población universitaria se tuvieron en cuenta los datos suministrados por admisiones y registros y Talento Humano. Con esta información se identificaron 6.964 estudiantes presenciales. Al total de la población estudiantil se agregó la población del personal administrativo, docente y de servicios generales, con un total de 953 personas, para un total de 7.917 personas. La población de estudiantes matriculados en carreras a distancia, no se contempló como valor total en estos cálculos.
Finalmente, la población que hace presencia diariamente en la universidad se calculó en 8000 personas. Haciendo un estimado de la población más la itinerante y el personal docente de catedráticos.

Con estos datos de población y conociendo la generación promedio de residuos sólidos por día en la universidad se calculó la generación de residuos per capita al día, mes y año.

Se identifica que una persona en el día puede producir 26,33 gr. aproximadamente. En el día podría no parecer un aporte significativo, sin embargo, al calcularlo en semanas y años, una persona en la universidad puede generar entre 158,02 y 184,36 gr. por semana. Y entre $7.901,25$ y $9.481,25$ gr. al año. Es decir una persona en la U.Q. genera de 7,9 a 9,5 kg de residuos año.

\section{Rutas de recolección interna}

Existen dos rutas de recolección interna en todas las dependencias, que son llevadas a cabo por dos diferentes operarios : Bienestar - Plantas Piloto y Ciencias Básicas Publicaciones.

Una ruta tiene como destino final el contenedor ubicado al frente de Ingeniería y la otra ruta hacia el contenedor cerca de la piscina.

Cada una de las rutas, tiene varias microrutas definidas como el recorrido en el cual el operario llena el carrito y lo lleva al sitio de acopio.

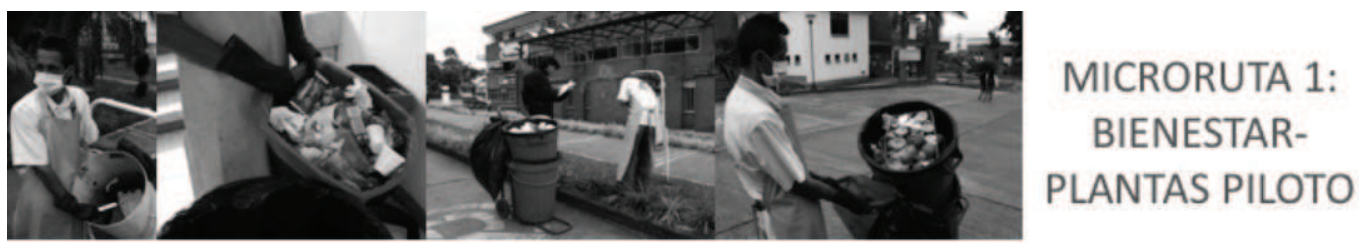

1.Bienestar Universitario, Ciencias de la salud, Puesto de frutas, CONTENEDOR

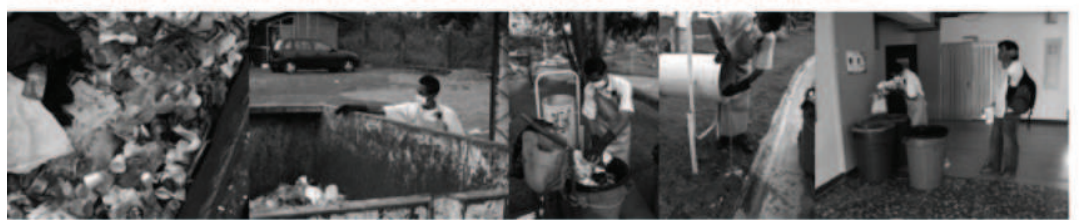

2.Capilla, Biblioteca, Cafetería Biblioteca, Audiovisuales, Auditorio, Salas de Internet, CONTENEDOR

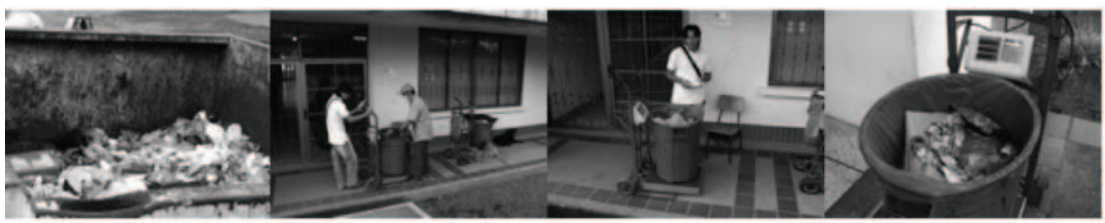

3. Ingeniería CONTENEDOR. 4. Educación, CONTENEDOR. 5. Economia, Agropecuaria, Plantas piloto, CONTENEDOR. 6.ESPECIALES

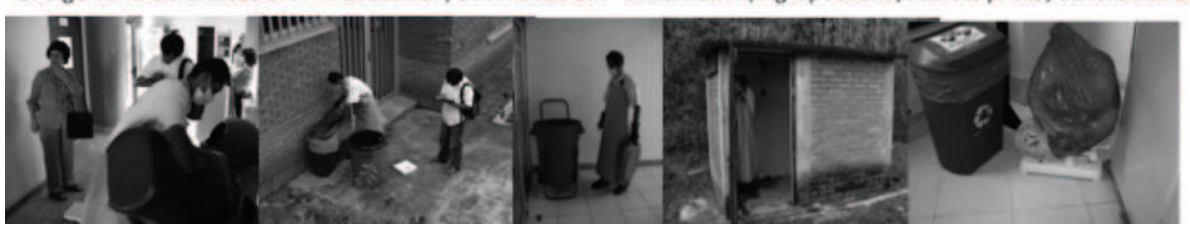

Figura 9 : Seguimiento a la ruta de recolección, pesaje de residuos

Rev. Invest. Univ. Quindio (20): 153 - 165. Armenia - Colombia 


\section{Tiempos de barrido y limpieza}

El seguimiento al trabajo de los operarios, su dibujo y posterior sistematización, nos permitió calcular los tiempos de barrido y limpieza que desempeña cada operario en cada una de las dependencias. Esta información permite identificar las áreas en $\mathrm{m}^{2}$ y longitudes en metros lineales que cubre un operario sobre determinado tiempo (horas), aportando a la identificación del tiempo y los recursos invertidos para la recolección de los residuos sólidos, según recomendación del RAS (Reglamento de Agua potable y Saneamiento).
Los datos de longitud de barrido y limpieza en relación con el tiempo de barrido y limpieza muestran que en el $50 \%$ de las dependencias un operario barre y limpia una longitud máxima de 2.024 metros lineales en 2,07 horas. $Y$ que en el $80 \%$ de las dependencias un operario barre y limpia una longitud máxima de 3.260 metros lineales en un máximo de 3 horas.

El análisis muestra que existe un rango de longitud de limpieza para las dependencias entre 1.162 y 2.887 metros lineales, con un intervalo de confianza del $95 \%$.

\section{CIENCIAS ECONOMICAS PRIMER PISO}

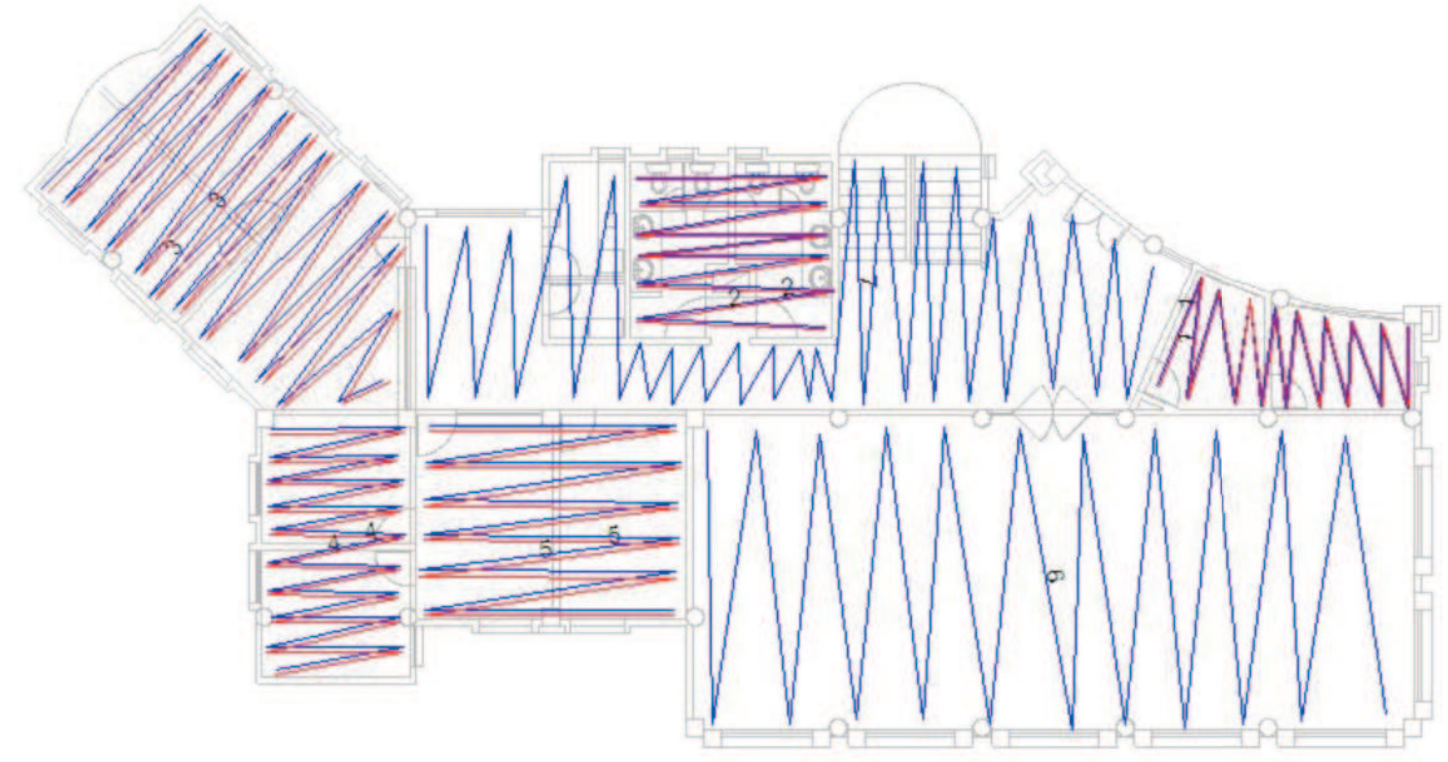

\begin{tabular}{|c|c|c|c|c|}
\hline $\begin{array}{c}\text { INIVTRSIDAD } \\
\text { DII. } \\
\text { QUINDIO }\end{array}$ & $\begin{array}{l}\text { Sitio de } \\
\text { estudio }\end{array}$ & 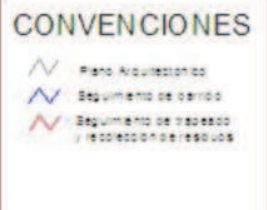 & 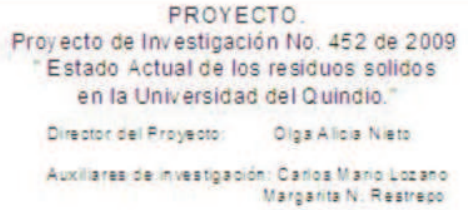 & 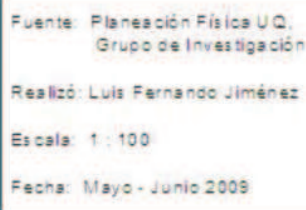 \\
\hline
\end{tabular}

Figura 10. Mapificación de áreas y longitudes de barrido y limpieza.

La figura 10, señala el registro del área y longitud recorrida por un operario en el primer piso del edificio de Ciencias Económicas, la línea azul muestra el recorrido en la actividad de barrido y la línea roja señala el recorrido para las actividades de trapeado, recolección de residuos sólidos, limpieza de polvo ("sacudido") y en algunos casos, actividades relacionadas con el servicio de cafetería.

\section{Impacto ambiental}

Los resultados nos señalan que la actividad más impactante es el acopio o disposición temporal en los contenedores, por la generación de malos olores, por no tener cubierta y porque allí se juntan todos los residuos, excepto los peligrosos y el papel.
Debido a que los contenedores se encuentran al aire libre, generan contaminación y malos olores, que molesta la población universitaria y aledaña al sector. En valor de impacto le siguen las actividades de recolección y transporte.

La inadecuada separación en la fuente, se refleja en las consecuencias que trae para la comunidad universitaria, en relación a los altos costos en los que la universidad debe incurrir para hacer su manejo.

El factor ambiental que se vió más afectado por el manejo de los residuos sólidos fue el aire en relación con los malos olores.

Identificación de las fuentes de agua cercanas, se reconoce

Rev. Invest. Univ. Quindío (20): 153 - 165. Armenia - Colombia 
la Quebrada la Aldana, que da origen a la microcuenca del mismo nombre, que alberga el fragmento de bosque de la Universidad del Quindío ubicado entre la carrera 15 y 19 del Municipio de Armenia. El nivel de afectación del sitio de acopio sobre la quebrada es mínimo, pues el escurrimiento de agua del contenedor es muy poca, está en área plana y se infiltra al suelo. El impacto identificado es de 3 , con unos máximos de 8 .

En la mesa del Sistema de Gestión Ambiental, se identifica que la afectación de la quebrada es por vertimientos líquidos, que vienen en su gran parte de una servidumbre que recibe las aguas residuales de la comuna 10 y en parte los vertimientos líquidos de la Universidad, no sólidos.

La revisión de las caracterizaciones previas sobre los vertimientos, Carga total vertida de $\mathrm{DBO}_{5}$ y SST, expresadas en Ton/mes, y los caudales medio diarios, producidos y vertidos expresado en Lt/seg, se está llevando a cabo por otros investigadores de la Facultad de Ingeniería, que hacen parte del Grupo del Sistema de Gestión Ambiental de la Universidad.

\section{Aspectos administrativos}

El manejo de residuos sólidos en la Universidad del Quindío, depende administrativamente de la Vicerrectoría Administrativa y Financiera, específicamente de la sección de Mantenimiento, que está a cargo del Jefe de Mantenimiento, quien calcula un $30 \%$ de su tiempo para la coordinación de los servicios de aseo.

El Jefe de Mantenimiento, tiene a su cargo para llevar a cabo este servicio a 8 personas de servicios generales que están en la planta de la Universidad. Y complementariamente con una Empresa que presta estos servicios, en la cual laboran 29 personas de servicios generales y una supervisora.

Por otra parte, realiza por licitación la contratación con la empresa prestadora de servicios para cubrir la demanda de servicios de aseo en la universidad. Actualmente lo ejecuta la empresa Telesai - Multiservir, con un grupo de 30 operarios.

\section{Consumo de servicios y tarifas}

Los servicios de recolección y disposición final, son prestados por SERVIGENERALES, empresa que facturó a la Universidad en el periodo enero - mayo 2009, $\$ 4.428,160$, es decir, un aproximado mensual de novecientos mil pesos (\$885.632), por un consumo estándar registrado en la factura de: $36,33 \mathrm{~m}^{3}$

Y la recolección y disposición final de residuos peligrosos clasificados como residuos peligrosos: biosanitarios y cortopunzantes), es contratada con EMDEPSA, este contrato establece un costo de $\$ 1600$ por kilo recolectado los días martes y sábado, con un estimado de producción de 200 $\mathrm{Kg} / \mathrm{mes}$. Es decir, $\$ 320.000$ mensuales

La universidad paga cerca de un millón doscientos mil pesos mensuales (\$1.205.000), por la recolección y disposición final de residuos sólidos. Proyectados al año tendremos catorce millones quinientos mil pesos anuales. (\$14.470.000).

Si bien el objetivo de este proyecto son los residuos sólidos, presentamos la información de los otros servicios en la Universidad, con miras al análisis general del Sistema de Gestión Ambiental.

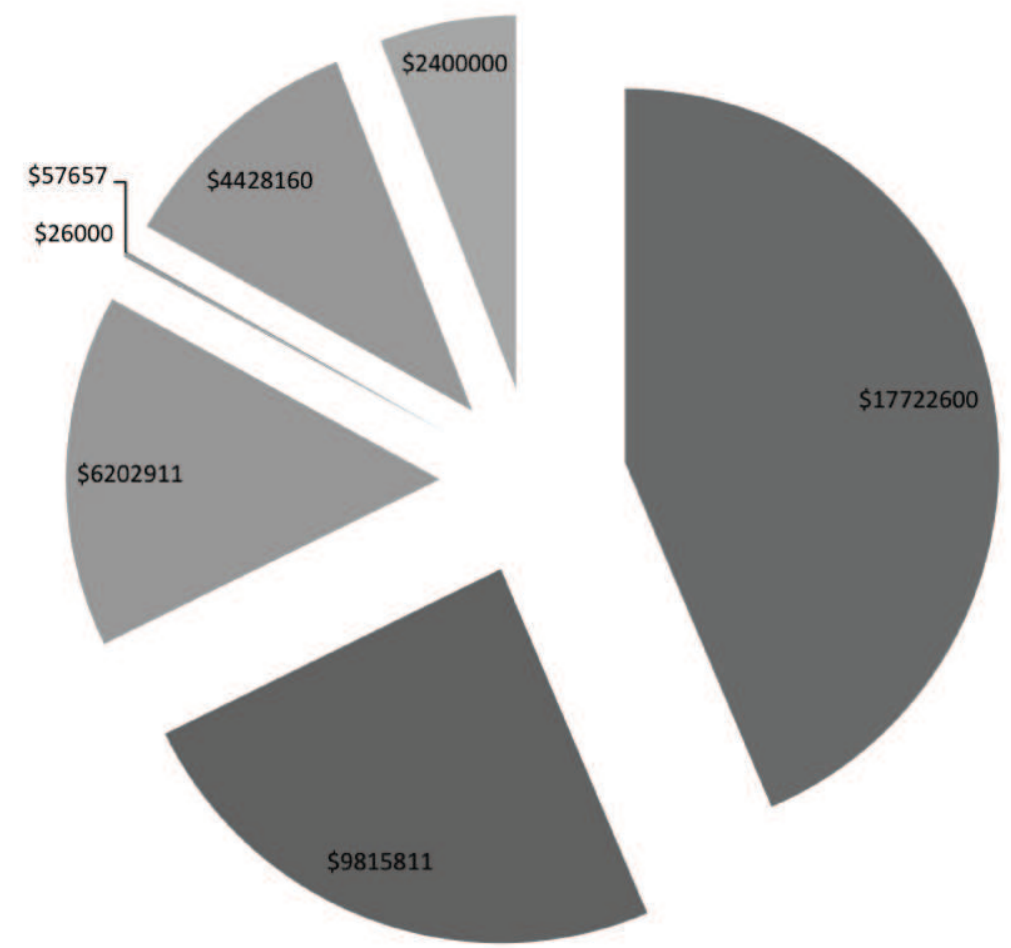

Energía

Ecueducto

Vertimientos

- Alcantarillado

Alumbrado público

" RECOLECCIÓN DE RESIDUOS SÓLIDOS GENERALES

- RECOLECCIÓN DE RESIDUOS SÓLIDOS ESPECIALES

Figura 11. Costo por pago de servicios en la universidad

Rev. Invest. Univ. Quindío (20): 153 - 165. Armenia - Colombia 
El mayor gasto en pago de servicios es en $\$ 17.722 .600$ en un período comprendido entre enero y mayo del presente año, con un consumo de $57.402 \mathrm{Kw}$. El servicio de energía es aportado por la EDEQ.

Es decir la Universidad paga aproximadamente $\$ 3.600 .000$ mensuales por el consumo de energía. Sin contar con el costo de alumbrado público, de $\$ 2.400 .000$ en el periodo de cinco meses, aproximadamente quinientos mil pesos mensuales, $(\$ 480.000)$.

Los pagos por el servicio de Acueducto se hacen a la EPA, con un consumo promedio de ( $13.666 \mathrm{~m} 3 / \mathrm{mes})$. En el período enero-mayo de 2009, el costo para la universidad es de $\$ 9.815 .811$ con una tasa ambiental de acueducto de $\$ 9.227$. Es decir el costo promedio mes por el servicio de acueducto es de $\$ 2.000 .000$ (\$1.963.000)

La Universidad pagó por vertimientos en este periodo $\$ 6.202 .011$, es decir un promedio mensual de $\$ 1.250 .000$. Y una proyección anual de $\$ 15.000 .000$, quince millones de pesos. Y una tasa ambiental de alcantarillado de $\$ 702.720$, es decir $\$ 150.000$, mensuales.

El menor gasto es para el servicio de alcantarillado (\$26.000), servicio en el cual la empresa prestadora (EPA), no discrimina consumos ni valores de cobro.

\section{Contactos con otros grupos}

Se estableció contacto con dos grupos de trabajo en el área de Gestión de Residuos sólidos, mediante la visita a cada una de sus sedes :

El de la Universidad Nacional, coordinado por el Dr. Luis H. Blanco, quien ofrece asesoría para el diseño del Sistema.

El de la Universidad Javeriana, liderado por el Profesor Camilo Contreras.

\section{Análisis de brecha}

Para hacer un adecuado análisis de brecha, debemos pensar en un escenario deseable para el manejo de residuos sólidos en la Universidad del Quindío.

La Universidad del Quindío tendrá durante los próximos dos años un Sistema de Gestión de Residuos Sólidos, basado en una estrategia de cultura ambiental, en el cual :

- Exista una política ambiental en la Universidad, que estimule el reuso, la recuperación y el reciclaje.

- En cada dependencia existan las canecas para una adecuada segregación en la fuente.

- La reutilización y reciclaje de papel se mida como un indicador de la cantidad de árboles que no se talan.

- Las rutas de recolección interna incluyan una ruta de biodegradables, con destino a la preparación de abono orgánico.

- El destino de final de productos no biodegradables obedezca a una política institucional de ciclo de vida de productos, por ejemplo en lámparas y tetrapack.

- Exista un único sitio de acopio interno, debidamente cubierto y manejado.

- Exista un convenio con asociación de recicladores para la clasificación y recuperación en el sitio de acopio.
- Disminuyan paulatinamente los residuos que se entregan a la empresa que los recoge para su disposición final y se entreguen compactados y densificados.

- Exista una estrategia de cultura ambiental que dinamice este proceso.

Buena parte de este escenario deseable, debe dibujarse en un Plan de Gestión de Residuos Sólidos-PGIRS.

\section{CONCLUSIONES}

La generación de residuos sólidos en la Universidad del Quindío es de $210 \mathrm{Kg} /$ día. Se calcularon unos máximos y mínimos, entre 10 a 12 meses año, es decir el año se generan entre 63 a 76 toneladas de residuos sólidos.

De acuerdo a la clasificación de residuos propuesta por el Decreto 2676 del 2000, la cantidad de residuos sólidos / día generados son los siguientes:

$\begin{array}{lrr}\text { Biodegradables u orgánicos } & 16,8 \mathrm{Kg} & 8 \% \\ \text { Reciclables (papel) } & 7,2 \mathrm{Kg} & 3,4 \% \\ \text { Ordinarios (incluye inertes) } & 182 \mathrm{Kg} & 86,66 \% \\ \text { Peligrosos (biosanitarios) } & 3,8 \mathrm{Kg} & 1,9 \%\end{array}$

En la Universidad se generan residuos sólidos peligrosos : biosanitarios y cortopunzantes, en medicina, bienestar universitario y biomédicas. La generación asciende al 1,9\% 3,8 kilos, respectivamente. Estos residuos tienen un manejo adecuado, según el marco normativo, en los guardianes y bolsas rojas. $Y$ son recogidos por Emdepsa para su disposición final. Los laboratorios de Aguas y de Suelos generan residuos químicos que son neutralizados debidamente y acopiados para su disposición final. No identificó la generación de residuos radioactivos.

Las dependencias que presentan mayor generación de residuos sólidos son: Ciencias básicas con $18 \%$ que corresponde a un peso de $37,69 \mathrm{~kg}$; Restaurante con $25 \%$ equivalente $53,127 \mathrm{~kg}$; Publicaciones $8,20 \%$ equivalente $17,26 \mathrm{~kg}$; Coliseo, Cafetería canchas y Piscinas 6,32\% equivalente 13,32 kg; Archivo 5,10\% equivalente 10,76 kg; Agroindustria 5,51\% equivalente $11,63 \mathrm{~kg}$; Educación 4,42\% equivalente $9,327 \mathrm{~kg}$; Ingeniería $4,17 \%$ equivalente $8,79 \mathrm{Kg}$; y Medicina 3,77\% equivalente 7,94 Kg.

La generación per cápita, es de 23,33 gr/día. En un año es de 8 a 9,5 kg per cápita. Se calculó identificando la población universitaria en 8.000 personas.

Existen 2 rutas de recolección interna, que funcionan diariamente y llevan los residuos sólidos a dos sitios de acopio : en la piscina y frente a ingeniería. Los residuos biosanitarios y cortopunzantes se acopian en un sitio dispuesto para tal fin en Biomédicas. La recolección de los residuos sólidos ordinarios la hace Servigenerales y la de los biosanitarios la lleva a cabo Emdepsa.

Se identifica el sitio de acopio, como punto crítico, porque allí se juntan todos los residuos : biodegradables, reciclables, inertes, excepto el papel y los biosanitarios. Y como principal impacto ambiental la contaminación 
atmosférica por olores y la presencia de algunos gallinazos. La contaminación hídrica en la microcuenca la Aldana por los residuos sólidos es mínima.

Si bien la Universidad tiene contratada con las organizaciones legalmente establecidas la recolección y disposición final de residuos sólidos ordinarios y biosanitarios, se está perdiendo la posibilidad de recuperar, reusar y reciclar buena parte de los residuos sólidos que genera, por lo tanto se propone un Sistema de Gestión de Residuos Sólidos, cuyo motor sea el Plan da Gestión Integral de Residuos Sólidos-PGIR.

\section{PROPUESTA PARA LA CONSTRUCCIÓN DE UN SISTEMA DE GESTIÓN AMBIENTAL EN LA UNIVERSIDAD DEL QUINDÍO}

Como parte del Sistema de Gestión Ambiental, este proyecto pretende dar aportes e iniciativas relacionadas con la problemática de los residuos sólidos dentro del campus.

De manera participativa se ha diseñado un Sistema de Gestión Ambiental que contempla:

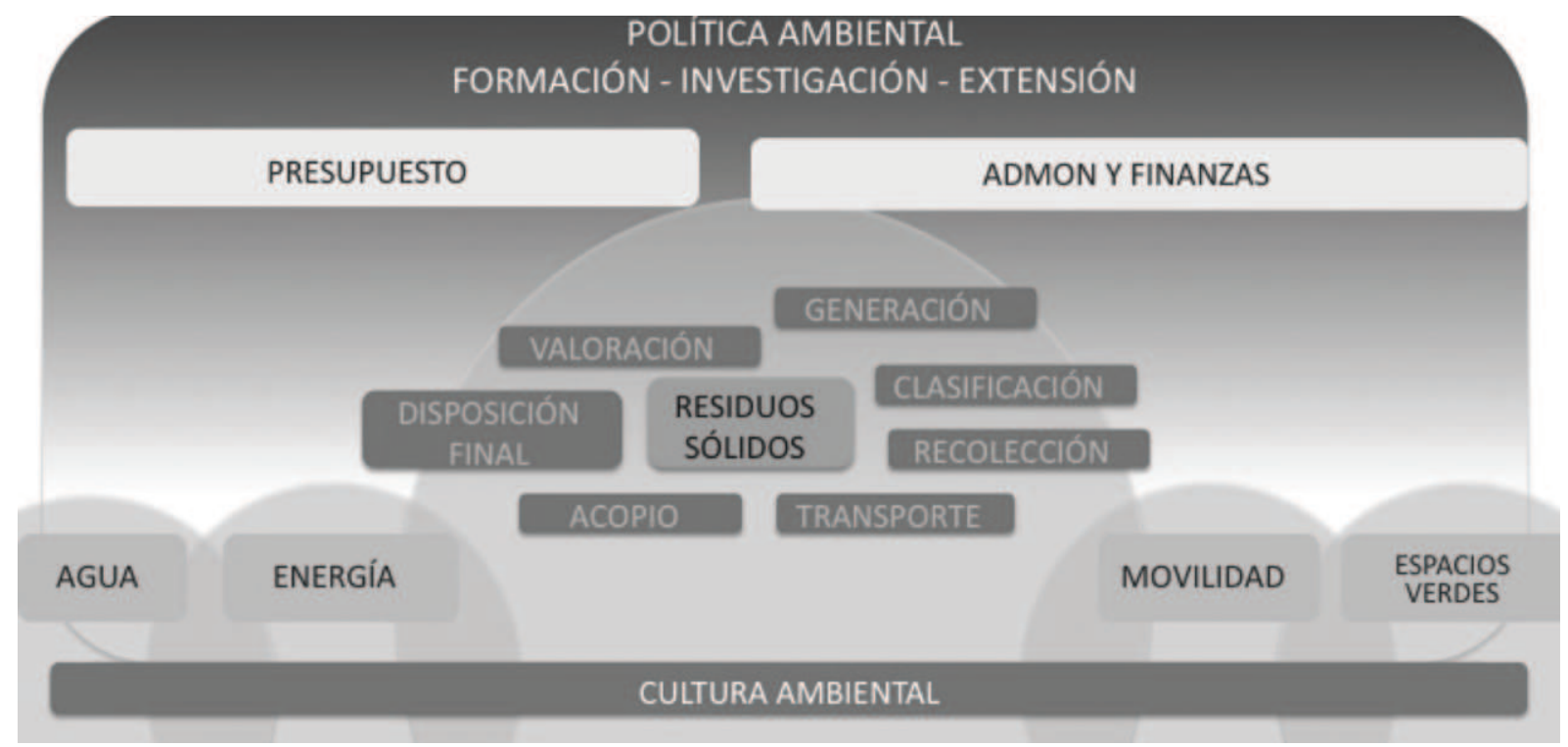

Figura 13. Sistema de Gestión Ambiental Universidad del Quindío

Una política ambiental (como una sombrilla) que oriente los procesos en la Universidad, una estrategia de cultura ambiental que moviliza esos procesos y un conjunto de subsistemas entre los cuales están espacios verdes (estructura ecológica), residuos sólidos, agua, energía, movilidad, aire. Dentro de estos subsistemas se encuentra el Sistema de Gestión de Residuos Sólidos.

\section{BIBLIOGRAFÍA}

- Administradora de riesgos profesionales. ARP de la Universidad del Quindío. (2006). Documentos de trabajo, no publicados. Armenia.

- Castro, A.L. García, G.E, Conde, L.M, Cuellar, J. (2006). “Producción más limpia en la Universidad del Quindío”. Proyecto de Grado. Ingeniería Civil. Armenia.

- Corporación Autónoma Regional del Quindío. CRQ. (2003). “Plan de Gestión Ambiental Regional”. PGAR. Armenia.

- Corporación Autónoma Regional del Quindío. CRQ. (2004). “Guía práctica para el manejo de residuos hospitalarios y similares". Armenia.

- Forero, S.M. y Ochoa, M.P. (2009). "Situación de la disposición final de residuos sólidos en Colombia - diagnóstico". Superintendencia delegada para acueducto, alcantarillado y aseo.

- Ministerio del Medio Ambiente. (2003). “Manual de procedimientos para la gestión integral de residuos hospitalarios y similares en Colombia MPGIR-H". Santafé de Bogotá. .

- Ministerio del Medio Ambiente. (2000). “Reglamento de Agua potable y saneamiento” - RAS. Santafé de Bogotá.

Rev. Invest. Univ. Quindío (20): 153 - 165. Armenia - Colombia 
- Ministerio de Salud. (2000). Decreto 2676 : “Gestión integral de los residuos hospitalarios y similares”. Santafé de Bogotá.

- Riveros, J. (2006). "Sistema de Gestión Ambiental podría ser modelo para Bogotá". Unimedios. UN Periódico. Sección medio ambiente. Santafé de Bogotá.

\section{AGRADECIMIENTOS}

Este proyecto se llevó a cabo con la financiación de la Vicerrectoría de Investigaciones, cuyo personal nos ha brindado permanente apoyo para su ejecución, a todos ellos queremos hacer un especial reconocimiento.

A la Vicerrectoría Administrativa y Financiera, el apoyo y buena voluntad de todas las personas que nos aportaron información.

A las Empresas Prestadoras de Servicios, que amablemente nos dedicaron tiempo para darnos información.

A las personas que hacen parte de la mesa del Sistema de Gestión Ambiental de la Universidad del Quindío.

Y especialmente a todas y cada una de las personas de servicios generales, cuyo trabajo realizado en la madrugada, cuando nadie está, nos permite vivir en unos espacios limpios y ordenados, sin percatarnos de su importancia.

Por su apoyo, colaboración y buena disposición para la realización de este trabajo.

A todos Muchas gracias.

Los investigadores 